\title{
More Robust Decode-and-Forward Relaying over Impulsive Noise Power Line Channels
}

\author{
Khaled M. Rabie*, Bamidele Adebisi*, Andrea M. Tonello $\ddagger$ and Galymzhan Nauryzbayev* \\ ${ }^{*}$ School of Engineering, Manchester Metropolitan University, Manchester, UK, M15 6BH \\ ${ }^{\ddagger}$ Institute of Networked and Embedded Systems, University of Klagenfurt, Klagenfurt 9020, Austria \\ ${ }^{\star}$ School of Engineering, Nazarbayev University, Astana, Kazakhstan \\ Email: \{k.rabie@mmu.ac.uk; b.adebisi@mmu.ac.uk; andrea.tonello@aau.at; galymzhan.nauryzbayev@nu.edu.kz\}
}

\begin{abstract}
This paper discusses the issue of energy-efficiency in power line communication (PLC) systems and introduces decode-and-forward (DF) energy-harvesting based relaying. The performance of the proposed system is analyzed in terms of the energy efficiency for which accurate analytical expressions are derived. To highlight the achievable gains, we also evaluate the performance of both the conventional DF relaying and the directlink systems. The presented simulated results clearly demonstrate the correctness of our analysis as well as the advantage of the proposed system over the conventional relaying and direct-link approaches. Additionally, it is shown that the proposed scheme will become more energy efficient as the harvested noise energy becomes larger.
\end{abstract}

Index Terms-Decode-and-forward relaying, energy efficiency, energy-harvesting, impulsive noise, log-normal fading, power line communication (PLC).

\section{INTRODUCTION}

$\mathbf{P}$ OWER line communication (PLC) technology has been one of the main enablers of numerous home-networking and smart grid applications [1], [2]. However, the high attenuation, frequency selectivity and the low transmit power restrictions remain the main challenges reducing the potential of this technology [3]-[5]. To reduce the severity of such challenges, various relaying protocols with different degrees of efficiency have been proposed for PLC systems. In particular, amplify-and-forward (AF) and decode-and-forward (DF) are the most widely investigated within the PLC research community. Although AF relaying is simple and easy to implement in practice, it was shown in [6] that it does not always enhance performance over the impulsive noise PLC channel compared to DF relaying.

In recent years, energy efficiency and power consumption performance in relaying PLC networks has received considerable amount of research interest and several attempts have been made by the scientific community towards this direction, see e.g. [7]-[9]. Although those studies have looked into various relaying scenarios with and without considering the static power (the power consumed by the PLC modem when no data is being transmitted), they all have focused on only optimizing the system parameters to reduce the transmit power. In contrast, our paper proposes to scavenge the high undesirable energy of impulsive noise over the PLC channel to further enhance energy efficiency. Specifically, we consider a dual-hop DF relaying system with time-switching relaying
(TSR) protocol for energy-harvesting at the relaying modem. This allows the relay to intelligently harvest the impulsive noise energy and then use it to forward the source information to the destination. The system performance will be evaluated in terms of energy efficiency. For the sake of comparison, the performance of the conventional DF relaying and the directlink schemes will also be presented.

The main contribution of this work resides in deriving analytical expressions of the energy efficiency for the three systems under consideration. Computer simulation are included to verify the accuracy of our analysis. In addition, to achieve best energy efficiency, extensive simulations are conducted solving the optimization problem of the energyharvesting time factor in several scenarios. For a more realistic power consumption scenario, we incorporate the dynamic power, static power and idle power of PLC modems in our power consumption profile; similar to [10] and [9]. The results reveal that the proposed system is able to offer remarkable energy efficiency enhancements when the end-to-end distance is sufficiently large. In addition, it is demonstrated that as the noise amplitude and/or its probability of occurrence increases, the achievable gains (relative to the conventional DF relaying and direct-link systems) will become more pronounced.

The rest of this paper is organized as follows. Section II, describes the system model. Section III analyzes the energy efficiency of the three systems under study. In Section IV, we present and discuss numerical examples and simulation results of the derived expressions. Finally, Section V concludes the paper.

\section{SySTEM MOdEL}

Fig. 1(a) shows a basic system diagram of the proposed system consisting of a source, relaying and destination PLC modems. The relay is based on DF protocol and is equipped with an energy harvester to scavenge the high impulsive noise energy; more details are provided below ${ }^{1}$. In addition, Figs. 1(b) and 1(c) illustrate the system diagrams of the conventional DF relaying and direct-link schemes.

The two DF relaying systems can be characterized using $h_{1}$ and $h_{2}$ representing the source-to-relay and relay-todestination channel gains with corresponding distances $d_{1}$ and

\footnotetext{
${ }^{1}$ The analysis for AF relaying PLC systems with energy-harvesting can be found in [11].
} 


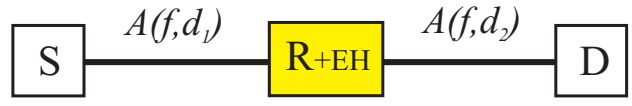

(a) EH-based DF relaying PLC system.

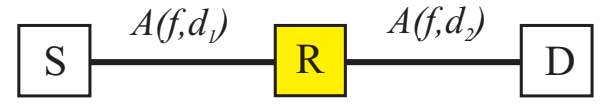

(b) Conventional DF relaying PLC system.

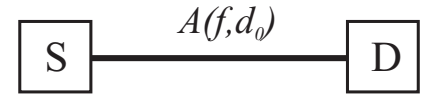

(c) Direct-link PLC system.

Figure 1: Basic system diagrams of the three systems under consideration. The letters S, R, D and EH denote the source, relay, destination and energyharvesting, respectively.

$d_{2}$, respectively; $h_{0}$ and $d_{0}=d_{1}+d_{2}$ denote the channel gain and the source-to-destination distance in the direct-link system, respectively. The channel gains of all links are assumed to be log-normally distributed [12], [13], and the distance and frequency dependent attenuation will be denoted as $A\left(f, d_{k}\right)$ where $f$ is the operating frequency and $k \in\{0,1,2\}$ is the link index.

The background noise $\left(n_{w}\right)$ and impulsive noise $\left(n_{i}\right)$ are modeled using the Bernoulli-Gaussian model [14] in which $n_{w}$ is complex Gaussian with variance $\sigma_{w}^{2}$ and $n_{i}$ is modeled as a Bernoulli-Gaussian random process with probability $p$ (this denotes the probability occurrence of impulsive noise). Note that the signal-to-background noise ratio (SBNR) and the signal-to-impulsive noise ratio (SINR) are defined respectively as $\mathrm{SBNR}=10 \log _{10}\left(1 / \sigma_{w}^{2}\right)$ and $\mathrm{SINR}=10 \log _{10}\left(1 / \sigma_{i}^{2}\right)$.

Three power consumption modes are adopted in this work, namely, dynamic power $\left(P_{d y n}\right)$, static power $\left(P_{s t c}\right)$ and idle power $\left(P_{i d l}\right)$, see [15] for more details. With this in mind, and assuming that all the PLC modems are identical and have same power consumption features, we can write the energy consumption for the proposed DF-EH system during phase I $\left(E_{t, 1}^{D F-E H}\right)$ and phase II $\left(E_{t, 2}^{D F-E H}\right)$, as

$$
E_{t, 1}^{D F-E H}=E_{t, 2}^{D F-E H}=\frac{(1-\tau) T}{2} \underbrace{\left(P_{d y n}+2 P_{s t c}+P_{i d l}\right)}_{P_{t, 1}^{D F-E H}=P_{t, 2}^{D F-E H}},
$$

where $P_{t, 1}^{D F-E H}$ and $P_{t, 2}^{D F-E H}$ represent the power consumption during phases I and II, respectively, whereas $T$ and $\tau$ will be defined below.

Similarly, we can express the total energy consumption for the conventional DF relaying and direct-link systems, respectively, as

$$
E_{t}^{D F}=T\left(P_{d y n}+2 P_{s t c}+P_{i d l}\right)
$$

and

$$
E_{t}^{D L}=T \underbrace{\left(P_{d y n}+2 P_{s t c}\right)}_{P_{t}^{D L}},
$$

where $P_{t}^{D L}$ is the power consumption in the direct-link system.

In the two DF relaying systems, we assume no directlink between the source and destination due to the high cable attenuation and therefore the end-to-end communication is accomplished over two phases. In this paper, we adopt TSR as an energy-harvesting protocol. Assuming that $T$ is the time required to transmit one block from the source to the destination, the energy-harvesting time will be given by $\tau T$ (the time used by the relaying PLC modem to harvest energy), where $0 \leq \tau \leq 1$ is referred to as the energyharvesting time factor. The remaining time $(1-\tau) T$ is used for information transmission, evenly for the two phases; i.e. $(1-\tau) T / 2$ for each phase. Note that the relaying modem has an external power source that provides $\left(P_{r e}\right)$, i.e. it is not entirely dependent on the harvested power $\left(P_{r h}\right)$ and that all the available relay power will be used to forward the information signal.

\section{Performance Analysis}

This section presents a step-by-step analytical derivation of the energy efficiency expressions for the three systems considered. To calculate the energy efficiency (bits/Hz/Joule), we first calculate the average spectral efficiency (bits/s/Hz) which, in non-Gaussian channels, can be calculated as [16]

$$
\Upsilon_{l}=(1-p) \mathbb{E}\left[\log _{2}\left(1+\gamma_{l}\right)\right]+p \mathbb{E}\left[\log _{2}\left(1+\frac{\gamma_{l}}{\beta_{l}}\right)\right],
$$

where $\gamma_{l ; l \in\{r, d\}}, \gamma_{l}$ is the SBNR at the receiving modem, $\beta=1+\sigma_{i}^{2} / \sigma_{w}^{2}$, and $r$ and $d$ denote the relay and destination modems. Note that $\beta=\beta_{r}=\beta_{d}$ in this case. The energy efficiency of the two links can then be calculated as

$$
\begin{aligned}
\rho_{r} & =\frac{\Upsilon_{r}}{P_{t, 1}^{D F-E H}}, \\
\rho_{d} & =\frac{\Upsilon_{d}}{P_{t, 2}^{D F-E H}} .
\end{aligned}
$$

The overall energy efficiency can now be determined by the minimum energy efficiency of the two links as follows [17]

$$
\rho=\min \left\{\rho_{r}, \rho_{d}\right\} .
$$

\section{A. EH-based DF Relaying PLC System}

To begin with, the received signal at the relay in the first phase can be expressed as

$$
y_{r}=\sqrt{P_{s}} A\left(f, d_{1}\right) h_{1} s+n_{r},
$$

where $P_{s}$ is the source PLC modem transmit power $\left(P_{d y n}^{S}\right), s$ is the information signal normalized as $\mathbb{E}\left[|s|^{2}\right]=1$ and $n_{r}$ is the noise signal at the relaying PLC modem with variance $\sigma_{r}^{2}$. It is assumed that all nodes are perfectly synchronized and no source information is transmitted during the energy-harvesting process at the relay. Hence, the harvested energy at the relay can be given by 


$$
E_{H}=\eta \tau T \sigma_{r}^{2}
$$

where $0<\eta<1$ is the energy-harvesting efficiency. Now, the received signal at the destination modem can be written as

$$
y_{d}=\sqrt{P_{r}} A\left(f, d_{2}\right) h_{2} \bar{s}+n_{d},
$$

where $\bar{s}$ is the decoded version of the source signal, $n_{d}$ is the noise at the destination modem with variance $\sigma_{d}^{2}$ and $P_{r}=$ $P_{r e}+P_{r h}$ is the relay total transmit power and $P_{r h}$ is given by

$$
P_{r h}=\frac{E_{H}}{(1-\tau) T / 2}=\frac{2 \tau \eta \sigma_{r}^{2}}{(1-\tau)} .
$$

Substituting (9) into (8) yields

$$
y_{d}=\sqrt{\frac{2 \tau \eta \sigma_{r}^{2}}{(1-\tau)}+P_{r e}} A\left(f, d_{2}\right) h_{2} \bar{s}+n_{d} .
$$

From (6) and (10), after grouping the information and noise terms, we can write the SBNRs at the relay and destination modems respectively as

$$
\gamma_{r}=\frac{P_{s} A\left(f, d_{1}\right)^{2} h_{1}^{2}}{\sigma_{r}^{2}}
$$

and

$$
\gamma_{d}=\frac{\left(2 \eta \tau \sigma_{r}^{2}+(1-\tau) P_{r e}\right) A\left(f, d_{2}\right)^{2} h_{2}^{2}}{(1-\tau) \sigma_{d}^{2}} .
$$

Using (4), the source-to-relay link's average spectral efficiency of the proposed system can be expressed as

$$
\Upsilon_{r}=\frac{(1-\tau)}{2} \sum_{i \in\{0,1\}} p_{i} \mathbb{E}\left[\log _{2}\left(1+\gamma_{r, i}\right)\right]
$$

where $\gamma_{r, 0}=\gamma_{r}$ and $\gamma_{r, 2}=\gamma_{r} / \beta$.

Equation (13) can be mathematically calculated as

$$
\Upsilon_{r}=\frac{(1-\tau)}{2} \sum_{i \in\{0,1\}} p_{i} \int_{0}^{\infty} \log _{2}(1+\gamma) f_{\gamma_{r, i}}(\gamma) d \gamma
$$

where $f_{\gamma_{r, 0}}(\cdot)$ and $f_{\gamma_{r, 1}}(\cdot)$ are the probability density functions (PDFs) of $\gamma_{r, 0}$ and $\gamma_{r, 1}$ at the relaying modem.

This can also be calculated using

$$
\Upsilon_{r}=\frac{(1-\tau)}{4 \ln (2)} \sum_{i \in\{0,1\}} p_{i} \int_{0}^{\infty} \frac{1-F_{\gamma_{r, i}}(\gamma)}{1+\gamma} d \gamma
$$

where $F_{\gamma_{r, 0}}(\cdot)$ and $F_{\gamma_{r, 1}}(\cdot)$ are the complementary distribution functions (CDFs) of $\gamma_{r, 0}$ and $\gamma_{r, 1}$, respectively.

Using (11), $F_{\gamma_{r, 0}}(\cdot)$ can be found as follows

$$
F_{\gamma_{r, 0}}(\lambda)=\operatorname{Pr}\left\{\frac{P_{s} A\left(f, d_{1}\right)^{2} h_{1}^{2}}{\sigma_{r}^{2}}<\lambda\right\}
$$

Hence,

$$
F_{\gamma_{r, 0}}(\lambda)=P_{r}\left\{h_{1}^{2}<\frac{\sigma_{r}^{2} \lambda}{P_{s} A\left(f, d_{1}\right)^{2}}\right\}
$$

Because $h_{1}^{2}$ has log-normal distribution, equation (17) can be expressed as

$$
F_{\gamma_{r, 0}}(\lambda)=\frac{1}{2}+\frac{1}{2} \operatorname{Erf}\left[\frac{\xi \ln (\lambda)-2 \mu_{1}-\xi \ln \left(\Xi_{0}\right)}{\sqrt{8} \sigma_{1}}\right]
$$

where $\xi=10 / \ln (10)$ is a scaling constant, $\Xi_{0}=$ $P_{s} A\left(f, d_{1}\right)^{2} / \sigma_{r}^{2}, \mu_{1}$ and $\sigma_{1}$ represent the mean and standard deviation of $10 \log _{10}\left(h_{1}\right)$, respectively.

Similarly, we have

$$
F_{\gamma_{r, 1}}(\lambda)=\frac{1}{2}+\frac{1}{2} \operatorname{Erf}\left[\frac{\xi \ln (\lambda)-2 \mu_{1}-\xi \ln \left(\Xi_{1}\right)}{\sqrt{8} \sigma_{1}}\right]
$$

where $\Xi_{1}=P_{s} A\left(f, d_{1}\right)^{2} / \sigma_{r}^{2} \beta$.

Substituting (18) and (19) into (15), we can calculate the spectral efficiency of at the relay as

$$
\begin{aligned}
\Upsilon_{r}=\frac{(1-\tau)}{4 \ln (2)} & \sum_{i \in\{0,1\}} p_{i} \int_{0}^{\infty} \frac{1}{1+\lambda}(1 \\
- & \left.\operatorname{Erf}\left[\frac{\xi \ln (\lambda)-2 \mu_{1}-\xi \ln \left(\Xi_{i}\right)}{\sqrt{8} \sigma_{1}}\right] d \lambda\right),
\end{aligned}
$$

Now, using substituting (20) into (4a) yields the energy efficiency of source-to-relay link. Furthermore, it is easy to show that the spectral efficiency of the relay-to-destination link can be calculated as

$$
\begin{aligned}
\Upsilon_{d}=\frac{(1-\tau)}{4 \ln (2)} & \sum_{i \in\{0,1\}} p_{i} \int_{0}^{\infty} \frac{1}{1+\lambda}(1 \\
- & \left.\operatorname{Erf}\left[\frac{\xi \ln (\lambda)-2 \mu_{2}-\xi \ln \left(\Lambda_{i}\right)}{\sqrt{8} \sigma_{2}}\right] d \lambda\right),
\end{aligned}
$$

where $\mu_{2}$ and $\sigma_{2}$ are the mean and the standard deviation of $10 \log _{10}\left(h_{2}\right)$, respectively; $\Lambda_{0}=\left(2 \eta \tau \sigma_{r}^{2}+(1-\tau) P_{r e}\right)$ $A\left(f, d_{2}\right)^{2} /(1-\tau) \sigma_{d}^{2}$ and $\Lambda_{1}=\Lambda_{0} / \beta$.

The energy efficiency of relay-to-destination link can now be determined by substituting (21) into (4b). Finally, the overall energy efficiency of the proposed system can be obtained by substituting $\rho_{r}$ and $\rho_{d}$ into (5). The solution of this will be found numerically using software tools.

\section{B. Conventional DF Relaying PLC System}

In the conventional DF system, the relaying modem has no energy-harvesting source and, hence, relies entirely on the external power supply. The energy efficiencies of the two links for this system can be straightforwardly written, respectively, as 


$$
\begin{aligned}
& \Upsilon_{r}=\frac{1}{4 \ln (2)} \sum_{i \in\{0,1\}} p_{i} \int_{0}^{\infty} \frac{1}{1+\lambda}(1 \\
&\left.-\operatorname{Erf}\left[\frac{\xi \ln (\lambda)-2 \mu_{1}-\xi \ln \left(\Xi_{i}\right)}{\sqrt{8} \sigma_{1}}\right] d \lambda\right),
\end{aligned}
$$

and

$$
\begin{aligned}
\Upsilon_{d}=\frac{1}{4 \ln (2)} & \sum_{i \in\{0,1\}} p_{i} \int_{0}^{\infty} \frac{1}{1+\lambda}(1 \\
- & \left.\operatorname{Erf}\left[\frac{\xi \ln (\lambda)-2 \mu_{2}-\xi \ln \left(\Gamma_{i}\right)}{\sqrt{8} \sigma_{2}}\right] d \lambda\right),
\end{aligned}
$$

where $\Gamma_{0}=P_{r e} A\left(f, d_{2}\right)^{2} / \sigma_{d}^{2}$ and $\Gamma_{1}=\Gamma_{0} / \beta$.

Substituting (22) and (23) into (4a) and (4b), respectively, then using (5), we will obtain the overall energy efficiency of the conventional DF relaying system.

\section{Direct-link PLC System}

For the direct-link system, the SBNR at the destination modem can be expressed as

$$
\gamma=\frac{P_{s} A\left(f, d_{1}+d_{2}\right)^{2} h_{0}^{2}}{\sigma_{d}^{2}}
$$

and the corresponding energy efficiency is calculated as

$$
\rho=\frac{\Upsilon}{P_{t}^{D L}}
$$

where $P_{t}^{D L}$ is defined in (3) and $\Upsilon$ is given by

$$
\begin{aligned}
\Upsilon=\frac{1}{2 \ln (2)} & \sum_{i \in\{0,1\}} p_{i} \int_{0}^{\infty} \frac{1}{1+\lambda}(1 \\
- & \left.\operatorname{Erf}\left[\frac{\xi \ln (\lambda)-2 \mu_{0}-\xi \ln \left(\Delta_{i}\right)}{\sqrt{8} \sigma_{0}}\right] d \lambda\right),
\end{aligned}
$$

where $\Delta_{0}=P_{s} A\left(f, d_{1}+d_{2}\right)^{2} / \sigma_{d}^{2}$ and $\Delta_{1}=\Delta_{0} / \beta$.

\section{RESUlts AND Discussions}

This section presents some numerical examples along with simulation results for the derived analytical expressions. The cable attenuation model used in our evaluations is $A(f, d)=$ $\exp \left(-\left(a_{o}+a_{1} f^{k}\right) d\right)$, where $f$ is the frequency in $\mathrm{MHz}, k$ is the exponent of the attenuation factor, $a_{o}$ and $a_{1}$ are constants determined from measurements [3]. In general, if not specified, we use the following setting: $P_{s}=P_{r e}=1 \mathrm{~W}, P_{\text {idle }}=0.1 \mathrm{~W}$, $\eta=1, p=0.01, f=30 \mathrm{MHz}, a_{0}=9.4 \times 10^{-3}, a_{1}=4.2 \times$ $10^{-7}, k=0.7$ and SBNR at all nodes is $25 \mathrm{~dB}$. In addition, we use $\mu_{k}=3 \mathrm{~dB}$ and $\sigma_{k}^{2}=4 \mathrm{~dB}$, where $k \in\{0,1,2\}$,

Fig. 2 shows the energy efficiency performance of the proposed system as a function of the energy-harvesting time factor for different noise scenarios. More specifically, Fig. 2a presents the performance when $p=0.01$ for SINR $=-10 \mathrm{~dB}$ and $-15 \mathrm{~dB}$ whereas Fig. $2 \mathrm{~b}$ demonstrates the results when

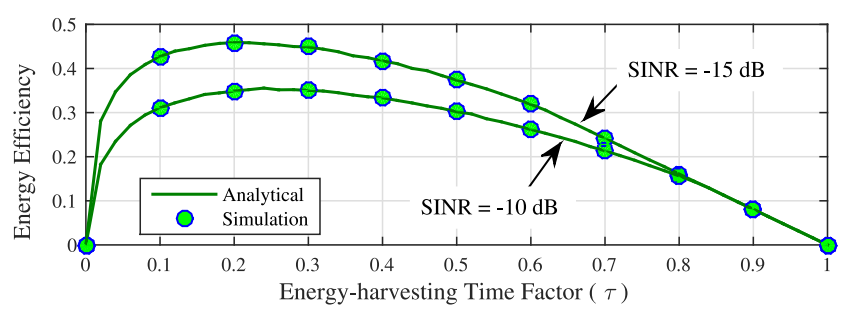

(a) $p=0.01$.

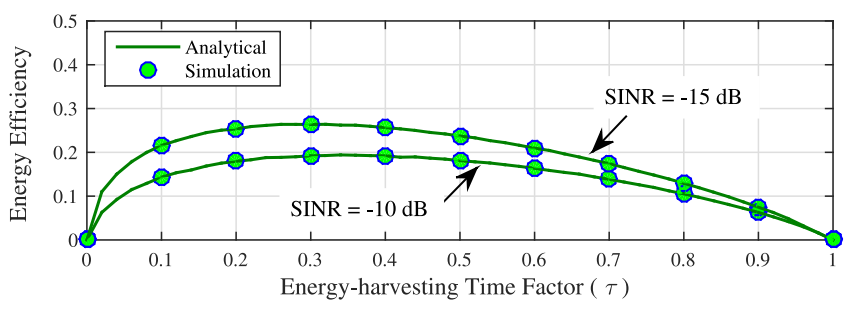

(b) $p=0.001$.

Figure 2: Energy efficiency performance as a function of the energyharvesting time factor for various noise probabilities and SINR values.

$p=0.001$ for the same SINR values. Note that in all these results, the relay is positioned midway between the source and destination modems, $d_{1}=d_{2}=50 \mathrm{~m}$ and $P_{s t c}=0.9 \mathrm{~W}$. In addition, it is assumed that $P_{r e}=0 \mathrm{~W}$; this is to more clearly illustrate the influence of the harvested energy on the performance of the proposed system. It can be seen from the two figures that as the noise energy becomes higher, i.e. SINR becomes smaller, the system tends to be more energy efficient. Also, comparing the two figures, it can be easily noticed that, for a given SINR value, the performance improves as $p$ is increased. This is simply justified by the fact that as either the noise amplitude or its probability of occurrence becomes higher, more energy can be harvested during the time $\tau$ at the relaying PLC modem. Another observation one can see is that the energy efficiency always deteriorates as $\tau$ approaches either zero or one. Therefore, optimizing this time factor is important to maximize the performance.

We now conduct extensive search for the optimal energyharvesting time factor and plot in Fig. 3 the corresponding maximum achievable energy efficiency of the optimized DFEH system with respect to the modem's static power for two end-to-end distances, namely, $300 \mathrm{~m}$ and $600 \mathrm{~m}$. Results for the conventional relaying and direct-link approaches are also included on this plot. These results are based on the following parameters $d_{1}=d_{0} / 3, p=0.01$ and $\operatorname{SINR}=-30 \mathrm{~dB}$. It is apparent from this figure that as the static power increases, the energy efficiency of the three systems degrades which is intuitive. It is also clear that when the end-to-end distance is large, i.e. $600 \mathrm{~m}$, the optimized DF-EH system always offers the best performance followed by the conventional DF approach whereas the direct-link system has the poorest performance. It is interesting to point out that this enhancement becomes more significant as the static power becomes smaller. On the other hand, however, when the end-to-end distance is relatively small, i.e. $300 \mathrm{~m}$ as in Fig. 3(b), the directlink system outperforms the other two relaying schemes. This implies that in short-distance scenarios, using multiple PLC 


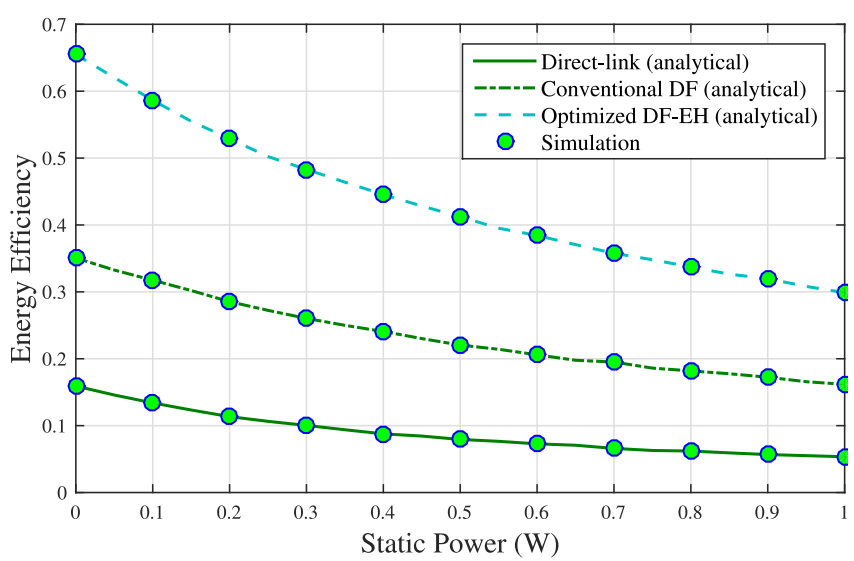

(a) $d=600 \mathrm{~m}$.

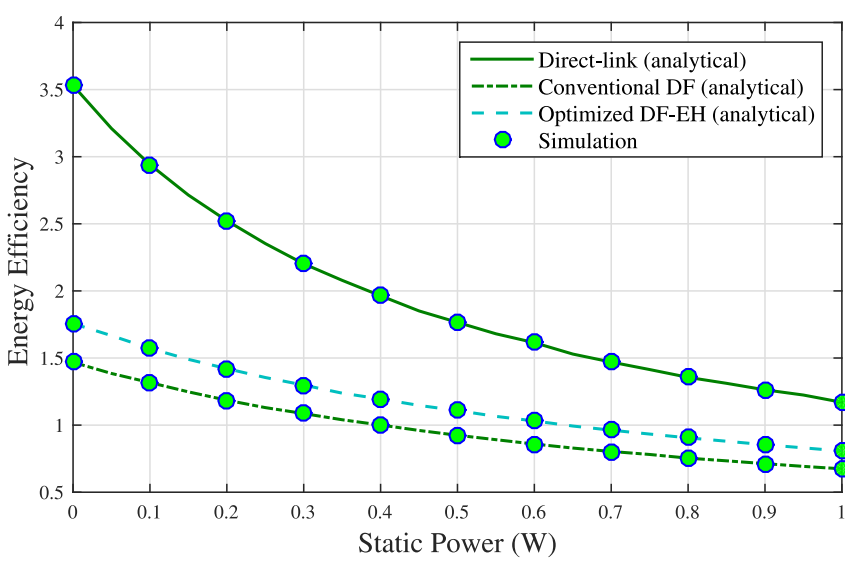

(b) $d=300 \mathrm{~m}$.

Figure 3: Energy efficiency performance versus the modem's static power for different end-to-end distances for the optimized DF-EH, conventional DF and direct-link systems.

modems can be energy inefficient due to the increased static power consumption. The final remark on these results is that, irrespective of the considered distance, the proposed system always has better performance than that of the conventional DF relaying system.

\section{CONCLUSION}

This paper analyzed the energy efficiency performance of a dual-hop relaying PLC system with energy-harvesting capabilities. In particular, DF relaying and TSR energy-harvesting protocols were implemented. The performance analyses of the conventional relaying and direct-link systems were also presented. For the three systems under consideration, we derived accurate analytical expressions of the energy efficiency. Results showed that as the impulsive noise energy becomes higher, more energy can be harvested at the relaying modem and, consequently, the proposed system becomes more energy efficient. It was also demonstrated that careful selection of the energy-harvesting time factor is vital to maximize the performance. Furthermore, results revealed that the optimized DF-EH system can considerably outperform the conventional relaying and direct-link schemes and this becomes more obvious when the end-to-end distance is relatively large. However, relaying in general was shown to be energy inefficient when the total distance is small in which case direct-link approach becomes more efficient.

\section{ACKNOWLEDGMENT}

This work was funded by Innovate UK as part of the "Smart In-building Micro Grid for Energy Management" (Innovate UK project 101836).

\section{REFERENCES}

[1] S. Galli, A. Scaglione, and Z. Wang, "For the grid and through the grid: The role of power line communications in the smart grid," Proc. IEEE, vol. 99, pp. 998-1027, Sept. 2011.

[2] A. Ikpehai, B. Adebisi, and K. M. Rabie, "Broadband PLC for clustered advanced metering infrastructure (AMI) architecture," Energies, vol. 9, pp. 1-19, Jul. 2016
[3] M. Zimmermann and K. Dostert, "A multipath model for the powerline channel," IEEE Trans. Commun., vol. 50, no. 4, pp. 553-559, 2002.

[4] C. Soltanpur, K. M. Rabie, B. Adebisi, and A. Wells, "Masreliezequalized VOFDM in non-gaussian channels: Power line communication systems," IEEE Systems Journal, vol. PP, no. 99, pp. 1-9, 2017.

[5] M. Rozman, A. Ikpehai, B. Adebisi, and K. M. Rabie, "Channel characterisation of cooperative relaying power line communication systems," in Proc. IEEE Int. Symp. Commun. Systems, Networks and Digital Signal Processing (CSNDSP), pp. 1-5, Jul. 2016.

[6] M. S. P. Facina, H. A. Latchman, H. V. Poor, and M. V. Ribeiro, "Cooperative in-home power line communication: Analyses based on a measurement campaign," IEEE Trans. Commun, vol. 64, pp. 778-789, Feb. 2016

[7] S. D'Alessandro, A. Tonello, and F. Versolatto, "Power savings with opportunistic decode and forward over in-home PLC networks," in Proc. IEEE Int. Symp. Power Line Commun. and Its Appl. (ISPLC), pp. 176181, Apr. 2011.

[8] S. D'Alessandro and A. M. Tonello, "On rate improvements and power saving with opportunistic relaying in home power line networks," $J$. Advances in Signal Process., vol. 2012, pp. 1-17, Sept. 2012.

[9] W. Bakkali, P. Pagani, and T. Chonavel, "Energy efficiency performance of relay-assisted power-line communication networks," in Proc. IEEE Consumer Commun. and Networking Conf. (CCNC), pp. 525-530, Jan. 2015.

[10] W. Bakkali, M. Tlich, P. Pagani, and T. Chonavel, "A measurementbased model of energy consumption for PLC modems," in Proc. IEEE Int. Symp. Power Line Commun. and Its Appl. (ISPLC), pp. 42-46, Mar. 2014.

[11] K. M. Rabie, B. Adebisi, and A. Salem, "Improving energy efficiency in dual-hop cooperative PLC relaying systems," in Proc. IEEE Int. Symp. Power Line Commun. and Its Appl. (ISPLC), pp. 196-200, Mar. 2016.

[12] A. Tonello, F. Versolatto, B. Bejar, and S. Zazo, "A fitting algorithm for random modeling the PLC channel," IEEE Trans. Power Del., vol. 27, pp. 1477-1484, Jul. 2012.

[13] A. M. Tonello, F. Versolatto, and A. Pittolo, "In-home power line communication channel: Statistical characterization," IEEE Trans. Commun., vol. 62, pp. 2096-2106, Jun. 2014.

[14] M. Ghosh, "Analysis of the effect of impulse noise on multicarrier and single carrier QAM systems," IEEE Trans. Commun., vol. 44, pp. 145147, Feb. 1996.

[15] K. M. Rabie, B. Adebisi, A. M. Tonello, and G. Nauryzbayev, "For more energy-efficient dual-hop DF relaying power-line communication systems," IEEE Systems Journal, vol. PP, no. 99, pp. 1-12, 2017.

[16] H. Vu, N. Tran, T. Nguyen, and S. Hariharan, "Estimating shannon and constrained capacities of Bernoulli-Gaussian impulsive noise channels in rayleigh fading," IEEE Trans. Commun., vol. 62, pp. 1845-1856, Jun. 2014.

[17] A. A. Nasir, X. Zhou, S. Durrani, and R. A. Kennedy, "Throughput and ergodic capacity of wireless energy harvesting based DF relaying network," in Proc. IEEE Int. Conf. Commun. (ICC), pp. 4066-4071, Jun. 2014. 